
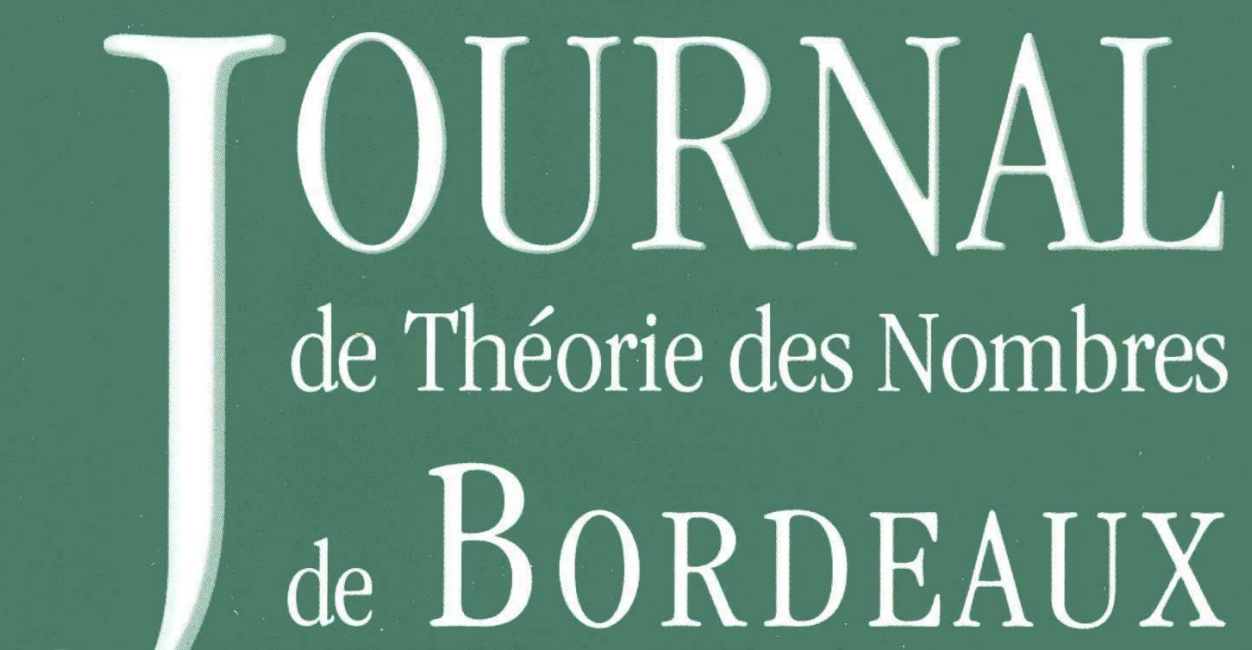

anciennement Séminaire de Théorie des Nombres de Bordeaux

\title{
Frédéric CHAPOTON
}

\section{Ramanujan-Bernoulli numbers as moments of Racah polynomials}

Tome 32, no 1 (2020), p. 205-215.

<http://jtnb.centre-mersenne.org/item?id=JTNB_2020__32_1_205_0>

(C) Société Arithmétique de Bordeaux, 2020, tous droits réservés. L'accès aux articles de la revue «Journal de Théorie des Nombres de Bordeaux » (http://jtnb.centre-mersenne.org/), implique l'accord avec les conditions générales d'utilisation (http://jtnb. centre-mersenne.org/legal/). Toute reproduction en tout ou partie de cet article sous quelque forme que ce soit pour tout usage autre que l'utilisation à fin strictement personnelle du copiste est constitutive d'une infraction pénale. Toute copie ou impression de ce fichier doit contenir la présente mention de copyright.

\section{cedram}

Article mis en ligne dans le cadre du

Centre de diffusion des revues académiques de mathématiques

http://www.centre-mersenne.org/ 


\title{
Ramanujan-Bernoulli numbers as moments of Racah polynomials
}

\author{
par FrÉDÉRIC CHAPOTON
}

RÉSUMÉ. Il est connu que les nombres de Bernoulli sont les moments d'une famille de polynômes orthogonaux. On obtient des énoncés semblables pour une autre suite de nombres rationnels, qui ont d'autres similarités avec les nombres de Bernoulli.

Abstract. The classical sequence of Bernoulli numbers is known to be the sequence of moments of a family of orthogonal polynomials. The same statement is obtained for another sequence of rational numbers, which is similar in many ways to the Bernoulli numbers.

\section{Introduction}

Let us consider the following sequence of rational numbers

$$
\begin{aligned}
\left(\mathscr{R}_{n}^{+}\right)_{n \geq 0}=1, \frac{1}{3}, \frac{1}{30},-\frac{1}{105}, \frac{1}{210},-\frac{1}{231}, \frac{191}{30030}, & \\
& -\frac{29}{2145}, \frac{2833}{72930},-\frac{140051}{969969}, \ldots
\end{aligned}
$$

and the almost identical companion sequence

$$
\begin{aligned}
\left(\mathscr{R}_{n}^{-}\right)_{n \geq 0}=1,-\frac{1}{6}, \frac{1}{30},-\frac{1}{105}, \frac{1}{210},-\frac{1}{231}, & \frac{191}{30030}, \\
& -\frac{29}{2145}, \frac{2833}{72930},-\frac{140051}{969969}, \ldots
\end{aligned}
$$

that differs only by the second term. These sequences are very close to the classical sequence of Bernoulli numbers, but not so well known.

The sequence $\mathscr{R}^{-}$seems to have first appeared, in a slightly implicit way and up to an easy power of 2 , in an article of Ludwig Seidel from 1877 [9], as the main diagonal of the difference table of the Bernoulli numbers. This diagonal is highlighted using a bold font in the table given there on page 181. Seidel proves that the two diagonals below and above the main diagonal in the same table are essentially given by the same sequence, up to multiplication by 2 .

Manuscrit reçu le 27 mai 2019, révisé le 13 septembre 2019, accepté le 25 octobre 2019.

2020 Mathematics Subject Classification. 33C45, 11B68, 11 Y65.

Mots-clefs. continued fraction, Bernoulli number, orthogonal polynomial. 
Later, the first terms of the sequence $\mathscr{R}^{-}$appeared explicitly in one of Srinivasa Ramanujan's notebooks (written between 1903 and 1914), as giving, up to a simple factor, the first coefficients in an unusual asymptotic expansion for the harmonic numbers into powers of the inverse of the triangular numbers $\left(\begin{array}{c}n+1 \\ 2\end{array}\right)$. This is displayed in Bruce Berndt's edition of Ramanujan's notebooks as the number (9) of [1, Ch. 38]). It can also be noted that the first term of this asymptotic expansion of Ramanujan was obtained by Ernesto Cesàro in 1885 in [2]. The first complete proof that the sequence $\mathscr{R}^{-}$describes this full asymptotic expansion was given by Mark Villarino in [11], where an historical account can be found.

The sequence $\mathscr{R}^{-}$has been considered again in 2005 by Kwang-Wu Chen [7], from a point of view close to that of Seidel. He obtained a functional equation and a continued fraction for their generating series. We will be more precise about his results later in Section 2 .

The sequence $\mathscr{R}^{+}$has recently surfaced in a very different algebraic context $[3,4]$ related to the notion of pre-Lie algebra. There is a complete algebraic theory of tree-indexed series, very similar to usual power series in one variable, but where monomials are indexed by finite rooted trees. These tree-indexed series can be multiplied (in a non-associative way) but also composed (in an associative way). One can therefore consider the group of tree-indexed series that are invertible for the composition. This group contains a special element $A$ with rational coefficients, which is a kind of tree-exponential and has very simple coefficients. Its inverse $\Omega$ has more subtle and interesting coefficients, among which the Bernoulli numbers $B_{n}$ for corollas and the numbers $\mathscr{R}_{n}^{+}$for another sequence of rooted trees.

After these works on tree-indexed series, it has been understood in [5] that the sequence $\mathscr{R}^{+}$also appears in the values at negative integers of some kind of non-standard $L$-function. More details will be given in Section 3.

The main aim of the present article, apart from advertising the sequences $\mathscr{R}^{+}$and $\mathscr{R}^{-}$, is to describe a new relationship between these sequences and the moments of some classical families of hypergeometric orthogonal polynomials, namely Racah polynomials (in some extended sense). This relationship in particular implies nice corollaries about continued fractions and Hankel determinants, by the general theory of orthogonal polynomials. We will not say more about this, because the exact statements can be easily reconstructed.

This is very similar to the known relationship between the Bernoulli numbers and another family of hypergeometric orthogonal polynomials, namely Hahn polynomials. This is therefore still another way in which the sequence $\mathscr{R}^{+}$is comparable with the Bernoulli numbers.

One word about terminology: there does not seem to be any accepted name for the sequences $\mathscr{R}^{-}$and $\mathscr{R}^{+}$. The name "median Bernoulli numbers" 
is used for the diagonal of the difference table of Bernoulli numbers, which differs from $\mathscr{R}^{-}$by powers of 2 . We propose that the name of "RamanujanBernoulli numbers" may be suitable for the sequence $\mathscr{R}^{-}$itself.

Let us end this introduction by some open questions.

First, it seems that the sequence $\mathscr{R}^{-}$alternates in sign. To the best of our knowledge, this is not yet proved. It is also expected that the associated non-standard $L$-function considered in Section 3 has a simple zero between consecutive negative integers.

There may exist $q$-analogues for some of the results of this article, in the spirit of the continued fractions for the $q$-Bernoulli numbers of Carlitz studied in [6], but they have so far remained elusive.

One can also wonder, in a very wild speculation, if there is, for the sequences $\mathscr{R}^{+}$or $\mathscr{R}^{-}$, something like the relationship between Bernoulli numbers and the algebraic K-theory of the ring of integers.

\section{Definitions}

1.1. Sequences $\mathscr{R}^{-}$and $\mathscr{R}^{+}$. Let us now give the formal definition of $\mathscr{R}^{-}$and $\mathscr{R}^{+}$.

Let us first introduce the classical Bernoulli numbers $B_{n}$, defined by

$$
\frac{t}{e^{t}-1}=\sum_{n \geq 0} B_{n} \frac{t^{n}}{n !} .
$$

Define a linear form $\Psi$ on the vector space $\mathbb{Q}[x]$ of polynomials in one variable $x$ with rational coefficients, by

$$
\Psi\left(x^{n}\right)=B_{n},
$$

for all $n \geq 0$.

The sequence $\mathscr{R}^{+}$is defined by

$$
\mathscr{R}_{n}^{+}=\Psi\left(\left(\begin{array}{c}
x+2 \\
2
\end{array}\right)^{n}\right),
$$

and the companion sequence $\mathscr{R}^{-}$by

$$
\mathscr{R}_{n}^{-}=\Psi\left(\left(\begin{array}{c}
x+1 \\
2
\end{array}\right)^{n}\right),
$$

for all $n \geq 0$. For example,

$$
\mathscr{R}_{6}^{+}=\frac{191}{30030}=1-\frac{9}{2}+\frac{49}{8}-\frac{157}{32}+\frac{8989}{2688}-\frac{157}{128}+\frac{245}{1408}-\frac{691}{174720} .
$$

and

$$
\mathscr{R}_{6}^{-}=\frac{191}{30030}=\frac{1}{2688}-\frac{1}{128}+\frac{25}{1408}-\frac{691}{174720} .
$$

The fact that $\mathscr{R}_{n}^{+}$and $\mathscr{R}_{n}^{-}$are the same except when $n=1$ follows from the next lemma. 
Lemma 1.1. For every $n \neq 1, \Psi\left(x^{n}(x+1)^{n}\right)=\Psi\left((x+1)^{n}(x+2)^{n}\right)$.

Proof. The case $n=0$ is trivial, so that one can assume $n \geq 2$. Let us compute the difference

$$
((x+1)(x+2))^{n}-(x(x+1))^{n}=2(x+1)^{n}\left(\sum_{\substack{k=0 \\
k \equiv 1(2)}}^{n}\left(\begin{array}{l}
n \\
k
\end{array}\right)(x+1)^{n-k}\right) .
$$

This is a linear combination of odd powers of $(x+1)$, excluding $x+1$. The image by $\Psi$ therefore vanishes, because the involved Bernoulli numbers are zero.

1.2. Racah's orthogonal polynomials. The orthogonal polynomials of Racah are defined by (see $[8, \S 1.2])$ :

$$
R_{n}(\lambda(x) ; \alpha, \beta, \gamma, \delta)={ }_{4} F_{3}\left(\begin{array}{c}
-n, n+\alpha+\beta+1,-x, x+\gamma+\delta+1 \\
\alpha+1, \beta+\delta+1, \gamma+1
\end{array}\right)
$$

where $\lambda(x)=x(x+\gamma+\delta+1)$ and using the standard notation for hypergeometric functions. The parameters $\alpha, \beta, \gamma$ and $\delta$ will remain implicit in all the notations.

A word of warning is required here. Racah polynomials are usually considered under the additional diophantine condition that

$$
\alpha+1=-N \quad \text { or } \quad \beta+\delta+1=-N \quad \text { or } \quad \gamma+1=-N
$$

for some integer $N \geq 0$. Under this condition, the polynomials $R_{n}$ are defined only for $n=0, \ldots, N$ and orthogonal with respect to a discrete finite measure. In this article, we will only consider parameters where the condition (1.6) does not hold. In this case, the polynomials $R_{n}$ can be defined for every $n \geq 0$, by the same two-term recurrence formula. One can therefore use this recurrence to define the linear form on the space of polynomials that plays the rôle of the measure. The fact that these polynomials are orthogonal under this linear form follows from the classical Favard's theorem, as stated for example in [10, Ch. 1, Th. 9]. Extending the usual meaning, one will still call them Racah polynomials.

Using the usual Pochhammer symbol $(x)_{k}=x(x+1) \cdots(x+k-1)$, the hypergeometric series above is given explicitly by the finite sum

$$
\sum_{k=0}^{n} \frac{(-n)_{k}(n+\alpha+\beta+1)_{k}(-x)_{k}(x+\gamma+\delta+1)_{k}}{(\alpha+1)_{k}(\beta+\delta+1)_{k}(\gamma+1)_{k} k !} .
$$

The polynomials $R_{n}$ are not monic in general. Setting

$$
R_{n}(x)=\frac{(n+\alpha+\beta+1)_{n}}{(\alpha+1)_{n}(\beta+\delta+1)_{n}(\gamma+1)_{n}} p_{n}(x),
$$

one obtains the corresponding family of monic orthogonal polynomials. 
As explained above, by the general theory of orthogonal polynomials and in particular by Favard's lemma, there exists a unique linear form $\Lambda$ on the vector space of polynomials in $x$ such that $\Lambda(1)=1$ and $\Lambda\left(p_{n}\right)=0$ for $n>0$. Then the moments of the family of orthogonal polynomials are given by $\Lambda\left(x^{n}\right)$ for $n \geq 0$.

Note that the linear form $\Lambda$ is also characterized by $\Lambda(1)=1$ and $\Lambda\left(R_{n}\right)=0$ for $n>0$. Hence there is no need to consider the monic orthogonal polynomials.

\section{Main theorems}

In this section, we will state five similar theorems, saying that some sequences are the sequences of moments of some specific families of Racah polynomials.

Theorem 2.1. The numbers $\left(2^{n} \mathscr{R}_{n}^{-}\right)_{n \geq 0}$ are the moments of the orthogonal polynomials $R_{n}$ of parameters $(\alpha, \beta, \gamma, \delta)=(0,-1 / 2,0,0)$.

Proof. We want to prove that

$$
\mu_{n}=\Lambda\left(x^{n}\right)=\Psi\left(x^{n}(x+1)^{n}\right)=2^{n} \mathscr{R}_{n}^{-},
$$

for all $n \geq 0$. By the characterisation of the linear form $\Lambda$, one just needs to check that $\Psi\left(R_{n}(x(x+1))\right.$ does vanish when $n>0$ and takes the value 1 at $n=0$. The second point is clear because $R_{0}=1$. We can use the explicit hypergeometric expression (1.7) at the given parameters:

$$
R_{n}(x(1+x))=\sum_{k=0}^{n} \frac{(-n)_{k}(n+1 / 2)_{k}(-x)_{k}(x+1)_{k}}{(1 / 2)_{k} k !^{3}} .
$$

Applying $\Psi$ and using Lemma A.1 (see the appendix) with parameters $(d, e, i, j)=(k, k, k-1, k)$ gives

$$
\sum_{k=0}^{n} \frac{(-n)_{k}(n+1 / 2)_{k}}{(1 / 2)_{k} k !(2 k+1)}={ }_{2} F_{1}\left(\begin{array}{c}
-n, n+1 / 2 \\
3 / 2
\end{array} ;\right),
$$

which is indeed 0 for every $n \geq 1$ by the Chu-Vandermonde identity.

We could get rid, in this theorem and the next four ones, of the factor $2^{n}$ in the sequences of moments, by scaling the variable in the orthogonal polynomials. Using the classical relationship between orthogonal polynomials, continued fractions and Hankel determinants (see for example [6]), one can deduce from the previous theorem (and similarly for the next four theorems) a nice continued fraction for the ordinary generating series of the sequence $\mathscr{R}^{-}$, and an explicit factorisation of the Hankel determinants made from $\mathscr{R}^{-}$. Both these results have been proved before by Kwang-Wu Chen in $[7, \S 5]$, without the connection with orthogonal polynomials. 
Theorem 2.2. The numbers $\left(2^{n} \mathscr{R}_{n+1}^{-} / \mathscr{R}_{1}^{-}\right)_{n \geq 0}$ are the moments of the orthogonal polynomials $R_{n}$ of parameters $(\alpha, \beta, \gamma, \delta)=(-1 / 2,1,0,0)$.

Proof. We want to prove that

$$
\mu_{n}=\Lambda\left(x^{n}\right)=\frac{1}{2 \mathscr{R}_{1}^{-}} \Psi\left(x^{n+1}(x+1)^{n+1}\right)=2^{n} \mathscr{R}_{n+1}^{-} / \mathscr{R}_{1}^{-},
$$

for all $n \geq 0$. By the characterisation of the linear form $\Lambda$, one just needs to compute

$$
\Psi\left(x(x+1) R_{n}(x(x+1))\right),
$$

whose value at $n=0$ is $2 \mathscr{R}_{1}^{-}$. We can use the explicit hypergeometric expression (1.7) at the given parameters:

$$
R_{n}(x(x+1))=\sum_{k=0}^{n} \frac{(-n)_{k}(n+3 / 2)_{k}(-x)_{k}(x+1)_{k}}{(1 / 2)_{k}(k+1) ! k !^{2}},
$$

and therefore

$$
x(x+1) R_{n}(x(x+1))=-\sum_{k=0}^{n} \frac{(-n)_{k}(n+3 / 2)_{k}(-x-1)_{k+1}(x)_{k+1}}{(1 / 2)_{k}(k+1) ! k !^{2}} .
$$

Applying $\Psi$ and using Lemma A.1 with parameters $(d, e, i, j)=(k+1, k+1$, $k-1, k)$ gives

$$
-\sum_{k=0}^{n} \frac{(-n)_{k}(n+3 / 2)_{k}}{(1 / 2)_{k} k !(2 k+3)(2 k+1)}=(-1 / 3){ }_{2} F_{1}\left(\begin{array}{c}
-n, n+3 / 2 \\
5 / 2
\end{array} ;\right),
$$

which is once again 0 for every $n \geq 1$ by the $\mathrm{Chu}$-Vandermonde identity.

Theorem 2.3. The numbers $\left(2^{n} \mathscr{R}_{n+1}^{+} / \mathscr{R}_{1}^{+}\right)_{n \geq 0}$ are the moments of the orthogonal polynomial $R_{n}$ of parameters $(\alpha, \beta, \gamma, \delta)=(0,1 / 2,0,-2)$.

Proof. We want to prove that

$$
\mu_{n}=\Lambda\left(x^{n}\right)=\frac{1}{2 \mathscr{R}_{1}^{+}} \Psi\left((x+1)^{n+1}(x+2)^{n+1}\right)=2^{n} \mathscr{R}_{n+1}^{+} / \mathscr{R}_{1}^{+},
$$

for all $n \geq 0$. By the characterisation of the linear form $\Lambda$, one just needs to compute

$$
\Psi\left((x+1)(x+2) R_{n}((x+1)(x+2))\right),
$$

whose value at $n=0$ is $2 \mathscr{R}_{1}^{+}$. We can use the explicit hypergeometric expression (1.7) at the given parameters:

$$
R_{n}(x(x-1))=\sum_{k=0}^{n} \frac{(-n)_{k}(n+3 / 2)_{k}(-x)_{k}(x-1)_{k}}{(-1 / 2)_{k} k !^{3}},
$$


and therefore

$$
\begin{aligned}
(x+1)(x+2) & R_{n}((x+1)(x+2)) \\
= & \sum_{k=0}^{n} \frac{(-n)_{k}(n+3 / 2)_{k}(x+1)(x+2)(-x-2)_{k}(x+1)_{k}}{(-1 / 2)_{k} k !^{3}} .
\end{aligned}
$$

Applying $\Psi$ and using Lemma A.2 (see the appendix) at $k$ gives

$$
-2 \sum_{k=0}^{n} \frac{(-n)_{k}(n+3 / 2)_{k}}{(-1 / 2)_{k} k !(2 k+3)(2 k+1)(2 k-1)}=(2 / 3)_{2} F_{1}\left(\begin{array}{c}
-n, n+3 / 2 \\
5 / 2
\end{array} ; 1\right)
$$

which is once again 0 for every $n \geq 1$ by the $\mathrm{Chu}$-Vandermonde identity.

Recall that $\mathscr{R}_{n}^{+}=\mathscr{R}_{n}^{-}$for every $n \geq 2$ by Lemma 1.1 . The two following statements therefore also hold with $\mathscr{R}^{-}$replacing $\mathscr{R}^{+}$.

Theorem 2.4. The numbers $\left(2^{n} \mathscr{R}_{n+2}^{+} / \mathscr{R}_{2}^{+}\right)_{n \geq 0}$ are the moments of the orthogonal polynomials $R_{n}$ of parameters $(\alpha, \beta, \gamma, \delta)=(-1 / 2,2,1,-1)$.

Proof. We want to prove that

$$
\mu_{n}=\Lambda\left(x^{n}\right)=\frac{1}{4 \mathscr{R}_{2}^{+}} \Psi\left((x+1)^{n+2}(x+2)^{n+2}\right)=2^{n} \mathscr{R}_{n+2}^{+} / \mathscr{R}_{2}^{+},
$$

for all $n \geq 0$. By the characterisation of the linear form $\Lambda$, one just needs to compute

$$
\Psi\left((x+1)^{2}(x+2)^{2} R_{n}((x+1)(x+2))\right),
$$

whose value at $n=0$ is $4 \mathscr{R}_{2}^{+}$. We can use the explicit hypergeometric expression (1.7) at the given parameters:

$$
R_{n}((x+1)(x+2))=\sum_{k=0}^{n} \frac{(-n)_{k}(n+5 / 2)_{k}(-x-1)_{k}(x+2)_{k}}{(1 / 2)_{k}(k+1) !^{2} k !}
$$

and therefore

$$
\begin{aligned}
(x+1)^{2} & (x+2)^{2} R_{n}((x+1)(x+2)) \\
& =-\sum_{k=0}^{n} \frac{(-n)_{k}(n+5 / 2)_{k}(x+1)(x+2)(-x-2)_{k+1}(x+1)_{k+1}}{(1 / 2)_{k}(k+1) !^{2} k !} .
\end{aligned}
$$

Applying $\Psi$ to this expression and using Lemma A.2 at $k+1$ gives

$$
2 \sum_{k=0}^{n} \frac{(-n)_{k}(n+5 / 2)_{k}}{(1 / 2)_{k} k !(2 k+1)(2 k+3)(2 k+5)}=(2 / 15){ }_{2} F_{1}\left(\begin{array}{c}
-n, n+5 / 2 \\
7 / 2
\end{array} ;\right),
$$

which is once again 0 for every $n \geq 1$ by the $\mathrm{Chu}$-Vandermonde identity. 
Theorem 2.5. The numbers $\left(2^{n} \mathscr{R}_{n+3}^{+} / \mathscr{R}_{3}^{+}\right)_{n \geq 0}$ are the moments of the orthogonal polynomials $R_{n}$ of parameters $(\alpha, \beta, \gamma, \delta)=(2,1 / 2,2,0)$ evaluated at $x-2$.

Proof. We want to prove that

$$
\mu_{n}=\Lambda\left(x^{n}\right)=\frac{1}{8 \mathscr{R}_{3}^{+}} \Psi\left((x+1)^{n+3}(x+2)^{n+3}\right)=2^{n} \mathscr{R}_{n+3}^{+} / \mathscr{R}_{3}^{+},
$$

for all $n \geq 0$. The linear form $\Lambda$ is characterized by the conditions

$$
\Lambda\left(R_{n}(x-2)\right)=0
$$

for all $n>0$ and 1 for $n=0$. We must therefore compute

$$
\Psi\left((x+1)^{3}(x+2)^{3} R_{n}((x+1)(x+2)-2)\right),
$$

whose value at $n=0$ is $8 \mathscr{R}_{3}^{+}$.

Because $x(x+3)=(x+1)(x+2)-2$, one can use the explicit hypergeometric expression (1.7) at the given parameters:

$$
R_{n}(x(x+3))=\sum_{k=0}^{n} \frac{(-n)_{k}(n+7 / 2)_{k}(-x)_{k}(x+3)_{k}}{(3 / 2)_{k}(3)_{k}(3)_{k} k !},
$$

and therefore

$$
\begin{aligned}
& (x+1)^{3}(x+2)^{3} R_{n}(x(x+3)) \\
& \quad=4 \sum_{k=0}^{n} \frac{(-n)_{k}(n+7 / 2)_{k}(x+1)(x+2)(-x-2)_{k+2}(x+1)_{k+2}}{(3 / 2)_{k}(k+2) !^{2} k !} .
\end{aligned}
$$

Applying $\Psi$ to this expression and using Lemma A.2 at $k+2$ gives

$$
-8 \sum_{k=0}^{n} \frac{(-n)_{k}(n+7 / 2)_{k}}{(3 / 2)_{k} k !(2 k+3)(2 k+5)(2 k+7)}=(-8 / 105)_{2} F_{1}\left(\begin{array}{c}
-n, n+7 / 2 \\
9 / 2
\end{array} 1\right) \text {, }
$$

which is once again 0 for every $n \geq 1$ by the Chu-Vandermonde identity.

Up to the same factor of $2^{n}$, the sequence of polynomials in the variable $u$ defined by

$$
\Psi\left(((x+u)(x+1-u))^{n}\right)=\Psi\left((x(x+1)+u(1-u))^{n}\right)
$$

can also be realised as a sequence of moments, by shifting by $u(1-u)$ the variable in the orthogonal polynomials used in Theorem 2.1. 


\section{Values of a non-standard $L$-function}

Let us consider the following analytic function

$$
L_{2}(s)=\sum_{n \geq 0} \frac{n+3 / 2}{\left(\begin{array}{c}
n+2 \\
2
\end{array}\right)} .
$$

This has been studied in [5], as the special case $P=\left(\begin{array}{c}x+2 \\ 2\end{array}\right)$ of the series

$$
L_{P}(s)=\sum_{n \geq 0} \frac{P^{\prime}(n)}{P(n)^{s}}
$$

attached to polynomials with no roots at positive integers.

The formula (3.2) is convergent in the right half-plane $\Re(s)>1$. It is known that $L_{P}$ admits an analytic continuation to a meromorphic function on the entire complex plane, with only a simple pole at 1 with residue 1 . Moreover, the values of $L_{P}$ at negative integers are rational numbers, given by

$$
L_{P}(1-n)=-\Psi\left(P^{n}\right) / n,
$$

for all integers $n \geq 1$. Here $\Psi$ is the linear form defined in Section 1.1.

Therefore, the numbers $\mathscr{R}_{n}^{+}$are closely tied with values of $L_{2}$ at negative integers:

$$
L_{2}(1-n)=-\mathscr{R}_{n}^{+} / n
$$

for all integers $n \geq 1$.

Let us conclude this section by a short remark on the analytic continuation of the functions $L_{P}$, already proved in [5]. Here we give another sketch of argument for the analytic continuation to a barely larger right half-plane, similar to a classical argument for the zeta function, and useful for numerical computations.

Let us define a polynomial $A(n)$ by the properties that $A(0)=1$ and

$$
A(n+1)-A(n)=P^{\prime}(n)
$$

for all $n \geq 0$. Then

$$
L_{P}(s)-\frac{1}{s-1}=\sum_{n=0}^{\infty} \frac{P^{\prime}(n)}{P(n)^{s}}-\int_{1}^{\infty} x^{-s} \mathrm{~d} x
$$

can be rewritten as

$$
\sum_{n=0}^{\infty} \int_{A(n)}^{A(n+1)} \frac{1}{P(n)^{s}} \mathrm{~d} x-\sum_{n=0}^{\infty} \int_{A(n)}^{A(n+1)} x^{-s} \mathrm{~d} x .
$$

Collecting the terms, one gets

$$
\sum_{n=0}^{\infty} \int_{A(n)}^{A(n+1)}\left(P(n)^{-s}-x^{-s}\right) \mathrm{d} x .
$$


The integral inside this sum can be rewritten as

$$
P(n)^{1-s} \int_{A(n) / P(n)}^{A(n+1) / P(n)}\left(1-y^{-s}\right) \mathrm{d} y .
$$

Let us assume that $P$ has degree $d$. Then $A$ has also degree $d$ and the same leading coefficient. Therefore both bounds in (3.8) tend to 1 as $n$ tends to infinity. The integral is therefore $O\left(n^{-2}\right)$ and the full term is $O\left(n^{d(1-s)-2}\right)$. It follows that the sum (3.7) is convergent when $\Re(s)>1-1 / d$.

\section{Appendix A. Evaluation lemmas}

This appendix contains two useful lemmas on the values of the linear form $\Psi$ on specific families of polynomials.

Lemma A.1. For all integers $d \geq 0, e \geq 0,0 \leq i \leq d-1$ and $0 \leq j \leq e$,

$$
\Psi\left(\left(\begin{array}{c}
-x+i \\
d
\end{array}\right)\left(\begin{array}{c}
x+j \\
e
\end{array}\right)\right)=(-1)^{d+e-i-j-1} \frac{1}{(d+e+1)\left(\begin{array}{c}
d+e \\
i+j+1
\end{array}\right)} .
$$

Proof. This follows directly from the known fact (see [5, Eq. (3.9)]) that, for all integers $0 \leq i \leq d$ and $0 \leq j \leq e$,

$$
\Psi\left(\left(\begin{array}{c}
x+i \\
d
\end{array}\right)\left(\begin{array}{c}
x+j \\
e
\end{array}\right)\right)=(-1)^{d+e-i-j} \frac{1}{(d+e+1)\left(\begin{array}{c}
d+e \\
d-i+j
\end{array}\right)} .
$$

Lemma A.2. For $k \geq 0$, there holds

$$
\Psi\left(\left(\begin{array}{c}
x+2 \\
2
\end{array}\right)\left(\begin{array}{c}
-x-3+k \\
k
\end{array}\right)\left(\begin{array}{c}
x+k \\
k
\end{array}\right)\right)=-\frac{1}{(2 k+3)(2 k+1)(2 k-1)}
$$

Proof. Note that the left-hand side of (A.3) is equal to

$$
(-1)^{k} \Psi\left(\left(\begin{array}{c}
x+2 \\
2
\end{array}\right)\left(\begin{array}{c}
x+2 \\
k
\end{array}\right)\left(\begin{array}{c}
x+k \\
k
\end{array}\right)\right) .
$$

Let us start by expanding the first product of binomials as

$$
\left(\begin{array}{c}
x+2 \\
2
\end{array}\right)\left(\begin{array}{c}
x+2 \\
k
\end{array}\right)=\sum_{2 \leq \ell \leq 4}\left(\begin{array}{c}
2 \\
\ell-2
\end{array}\right)\left(\begin{array}{c}
k \\
\ell-2
\end{array}\right)\left(\begin{array}{l}
x+\ell \\
2+k
\end{array}\right),
$$

by a general formula (see [5, Eq. (2.2)]). Then (A.4) becomes

$$
(-1)^{k} \sum_{2 \leq \ell \leq 4}\left(\begin{array}{c}
2 \\
\ell-2
\end{array}\right)\left(\begin{array}{c}
k \\
\ell-2
\end{array}\right) \Psi\left(\left(\begin{array}{c}
x+\ell \\
2+k
\end{array}\right)\left(\begin{array}{c}
x+k \\
k
\end{array}\right)\right)
$$


which can be evaluated using (A.2) as

$$
\sum_{2 \leq \ell \leq 4}(-1)^{\ell} \frac{\left(\begin{array}{c}
2 \\
\ell-2
\end{array}\right)\left(\begin{array}{c}
k \\
\ell-2
\end{array}\right)}{(3+2 k)\left(\begin{array}{c}
2+2 k \\
\ell
\end{array}\right)} .
$$

This can be expanded and simplified into the right-hand side of (A.3).

\section{References}

[1] B. C. Berndt, Ramanujan's notebooks. Part V, Springer, 1998, xiv+624 pages.

[2] E. Cesìro, "Sur la série harmonique.", Nouv. Ann. IV (1885), p. 295-296.

[3] F. Снаротоn, "A rooted-trees $q$-series lifting a one-parameter family of Lie idempotents", Algebra Number Theory 3 (2009), no. 6, p. 611-636.

[4] - "Sur une série en arbres à deux paramètres", Sémin. Lothar. Comb. 70 (2013), article ID B70a (20 pages).

[5] F. Chapoton \& D. Essouabri, " $q$-Ehrhart polynomials of Gorenstein polytopes, Bernoulli umbra and related Dirichlet series", Mosc. J. Comb. Number Theory 5 (2015), no. 4, p. 1338.

[6] F. Chapoton \& J. Zeng, "Nombres de q-Bernoulli-Carlitz et fractions continues", J. Théor. Nombres Bordeaux 29 (2017), no. 2, p. 347-368.

[7] K.-W. Chen, "A summation on Bernoulli numbers", J. Number Theory 111 (2005), no. 2, p. $372-391$.

[8] R. Kоекоек \& R. Swartтouw, "The Askey-scheme of hypergeometric orthogonal polynomials and its q-analogue", Tech. report, Delft Univ. of Technology, 1994, http://homepage . tudelft.nl/11r49/documents/as98.pdf.

[9] P. L. SEIDEL, "Ueber eine einfache Entstehungsweise der Bernoulli'schen Zahlen und einiger verwandten Reihen", Sitzungsberichte der mathematisch-physikalischen Classe der $k$. $b$. Akademie der Wissenschaften zu München 7 (1877), p. 157-187, http://publikationen. badw.de/de/003384831.pdf.

[10] G. Viennot, "Une théorie combinatoire des polynômes orthogonaux", Lecture Notes UQAM, 219 pages, 1984, http://www.xavierviennot.org/xavier/polynomes_ orthogonaux.html.

[11] M. B. Villarino, "Ramanujan's harmonic number expansion into negative powers of a triangular number", JIPAM, J. Inequal. Pure Appl. Math. 9 (2008), no. 3, article ID 89 (12 pages).

Frédéric CHAPOTON

Institut de Recherche Mathématique Avancée

UMR 7501, Université de Strasbourg et CNRS

7 rue René Descartes

67000 Strasbourg, France

E-mail: chapoton@unistra.fr 